glassy flask-shaped cell at the base of which is a layer of pigment with a nucleus embedded in it (Fig. 1).

The presence of the glassy cells together with the pigmented layer facing outwards is significant, and seems to point to a visual function for the papillæ, at least in so far as this species is concerned. It also supports Whitman's assertion that the eyes and the segmental papillæ were, originally, morphological as well as physiological equivalents.

Department of Zoology,

M. L. Bhatia

University of Delhi,

Delhi 8.

April 21.

1 Whitman, C. O., Proc. Amer. Acad. Arts and Sciences (1885).

2 Bhatia, M. L., Ind. Zool., Mem. 8 (1941).

\section{Antigens and Mating Types in Escherichia coli}

EVIDENCE has been offered by one of us $^{1}$ for the existence of properties correlated with sex compatibility in Escherichia coli. According to these data, sex-incompatible $(F-)$ strains have a lower acid agglutination point and a far greater stability of broth-cultures as compared with $F+$ strains of corresponding phenotype. These and other related properties of cell surface and the $F+$ state are transmitted infectively from $F+$ to $F-$ strains at the same time. A search for an antigenic equivalent of such properties was but an obvious implication of these findings.

Some strains of Escherichia coli $K 12$ employed in previous researches were used for preparing immune sera by inoculating rabbits intravenously. Two phenotypes were chosen, one of which is defined by biotin and methionine deficiency and resistance to the bacteriophage $T_{1}$, and the other by threonine, leucine and thiamine deficiency and inability to ferment lactose, maltose, galactose, arabinose, xylose and mannitol. The $F-$ and the $F+$ variants of each phenotype were used.

Both $O$ and $K$ antigens were prepared from each strain, aiming at providing evidence for the somatic or the capsular nature of the antigenic structure, if any, correlated with sex compatibility. The $O$ antigen was prepared by heating young broth-cultures at $100^{\circ} \mathrm{C}$. for $2 \frac{1}{2} \mathrm{hr}$. The thermolabile $K$ antigen was prepared by suspending in saline smooth opaque colonies freshly grown on nutrient agar supplemented with 1 per cent glucose. Rabbits were injected at 4-day intervals and sera collected when testbleedings revealed an agglutinating titre of about $1: 1,000$.

Fresh suspensions in saline of the four strains, that is, the two mating types of the two phenotypes, were used for the agglutination reactions. For this purpose cultures were used at the logarithmic growth stage under conditions which were optimal for both recombination and $F$ infection.

The results of the agglutination reactions showed that : (1) anti- $O$ sera give no appreciable agglutination with any strain unless the cells are boiled for $2 \frac{1}{2}$ hr. ; (2) anti- $K$ sera differentiate $F-$ from $F+$ untreated cells of the same phenotype in that they agglutinate $F-$ much more rapidly and in larger clumps than they do $F+;(3)$ the phenotype and the $F$ state of the antigen used for injecting rabbits do not affect appreciably the type of antibody pro. duced, but the anti- $K$ sera have a higher titre than the anti- $O$ ones ; (4) controls, either in the absence of immune sera or with normal serum from untreated rabbits, are fully negative; (5) the Kauffmann serum $O 26$ B6 (kindly supplied by Dr. A. Buogo) gives, with the same antigens, a reaction identical with that of our anti- $K$ sera.

These observations seem to indicate that the antigenic difference, if any, between $F+$ and $F$ - cells of the same phenotype is to be understood as a difference in surface antigens, which is in good agreement with our previous results. Possibly, $F+$ cells are deprived of, or $F$ - cells are more abundantly provided with, a capsular antigen which seems to be of the thermolabile $B$ type.

On the basis of these preliminary results, wider researches have been undertaken in order to: $(a)$ analyse, qualitatively and quantitatively, the antigenic structure of Escherichia coli strains belonging to different mating types; $(b)$ ascertain the effect of adsorbed antibodies on the kinetics of recombination and $F$ infection.

Moreover, it is apparent that if $F$ infection is substantiated, at least partially, by transformation of antigenic properties, these researches might also reinforce the interpretation of the well-known phenomenon of paragglutination ${ }^{2}$.

Istituto di Patologia Generale,

G. A. Maccacaro C. Colombo

Università di Milano. April 25.

'Maccacaro, G. A., Nature, 176, 125 (1955). Maccacaro, G. A., and

Comolli, $\mathbf{R}, J$ Gen Microbiol, (in the press).

${ }^{2}$ Zironi, A., Atti Mem. Soc. Lomb. Med., 6, 555 (1938).

\section{5-Hydroxytryptamine in Scorpion Venom}

IN the course of an investigation of the toxic factors in the venom of Leiurus quinquestriatus $(\mathrm{H}$. and $\mathrm{E}$., 1829), one of the constituents of the venom has been found to be 5-hydroxytryptamine.

The venom was obtained by electrical stimulation of the telson ${ }^{1}$, and was dried in vacuo and dissolved in water when required. The 5-hydroxytryptamine was identified by paper chromatography, developing with three different solvent systems and using the colour reactions with $p$-dimethylaminobenzaldehyde or diazotized $p$-nitroaniline, or the fluorescence produced after treatment with potassium dichromate and formalin (ef. Erspamer ${ }^{2}$ ).

Comparison of the areas of the spots with those from known concentrations of 5 -hydroxytryptamine suggested concentrations of 3-4 $\mu \mathrm{gm}$. 5-hydroxytryptamine base/mgm. dry venom. Assays of the crude venom on the rat uterus preparation or (with less precision) on guinea pig ileum confirmed these concentrations, and the activity of the venom on these preparations could be completely antagonized by dihydroergotamine. Assays of eluates of 5-hydroxytryptamine from chromatograms of the venom gave rather lower values, 1-2 $\mu \mathrm{gm} . / \mathrm{mgm}$. venom; but eluates from other areas of the chromatogram did not show any activity on these preparations.

These concentrations of 5 -hydroxytryptamine are even higher than those reported in wasp venom ${ }^{3}$, and may contribute to the pain resulting from a sting. It is of interest that Mohammed and El Karemi ${ }^{4}$ have claimed that a combination of dihydroergotamine and atropine, both capable of antagonizing 